\title{
A Patch Antenna Design for Application in a Phased-Array Head and Neck Hyperthermia Applicator
}

\author{
Margarethus M. Paulides*, Jurriaan F. Bakker, Nicolas Chavannes, Member, IEEE, and \\ Gerard C. Van Rhoon, Member, IEEE
}

\begin{abstract}
In this paper, we describe a specifically designed patch antenna that can be used as the basis antenna element of a clinical phased-array head and neck hyperthermia applicator. Using electromagnetic simulations we optimized the dimensions of a probe-fed patch antenna design for operation at $433 \mathrm{MHz}$. By several optimization steps we could converge to a theoretical reflection of $-38 \mathrm{~dB}$ and a bandwidth $(-15 \mathrm{~dB})$ of $20 \mathrm{MHz}$ $(4.6 \%)$. Theoretically, the electrical performance of the antenna was satisfactory over a temperature range of $15{ }^{\circ} \mathrm{C}-35^{\circ} \mathrm{C}$, and stable for patient-antenna distances to as low as $4 \mathrm{~cm}$. In an experimental cylindrical setup using six elements of the final patch design, we measured the impedance characteristics of the antenna 1) to establish its performance in the applicator and 2) to validate the simulations. For this experimental setup we simulated and measured comparable values: $-21 \mathrm{~dB}$ reflection at $433 \mathrm{MHz}$ and a bandwidth of $18.5 \mathrm{MHz}$. On the basis of this study, we anticipate good central interference of the fields of multiple antennas and conclude that this patch antenna design is very suitable for the clinical antenna array. In future research we will verify the electrical performance in a prototype applicator.
\end{abstract}

Index Terms-Antenna design, FDTD model, hyperthermia, patch antenna.

\section{INTRODUCTION}

I N PREVIOUS publications [1], [2] we have explained the need for a site-specific design of an applicator that will adequately heat head and neck ( $\mathrm{H} \& \mathrm{~N})$ tumors. In an exploratory theoretical study [2] we demonstrated that such a site-specific applicator must consist of multiple antenna elements to provide adequate control of the specific absorption rate (SAR) deposition pattern. We also showed that $433 \mathrm{MHz}$ (ISM frequency in Europe: a frequency allocated for industry, science and medicine) is a suitable frequency for heating H\&N tumors with a multi-antenna applicator. In this paper, we will address the

Manuscript received September 13, 2006; revised February 9, 2007. This work was supported by the Maurits en Anna de Kock foundation and the Dutch Cancer Society under Grant DDHK 2003-2855. Asterisk indicates corresponding author.

*M. M. Paulides is with the Erasmus MC-Daniel den Hoed Cancer Center, Department of Radiation Oncology, Section Hyperthermia, P.O. Box 5201, NL-3008 AE, Rotterdam, The Netherlands (e-mail: m.paulides@erasmusmc.nl).

J. F. Bakker and G. C. Van Rhoon are with the Erasmus MC-Daniel den Hoed Cancer Center, Department of Radiation Oncology, Section Hyperthermia, NL-3008 AE, Rotterdam, The Netherlands.

N. Chavannes is with Schmid and Partner Engineering AG (SPEAG), CH-8004 Zurich, Switzerland.

Digital Object Identifier 10.1109/TBME.2007.895111 first step of the applicator design, i.e., the design of a single antenna element.

The problems involved with hyperthermia antenna design are very different from the design of antennas in free space and should not be underestimated [3]. First, the antenna radiates into a lossy medium, which has a considerable influence on the impedance, bandwidth, and antenna losses [4], [5]. For this reason, the antenna design procedures described in the literature could not be used, since these were developed for a lossless environment (usually air) and simulations have to be verified by measurements. Second, the presence of a patient in the near field can also have considerable impact on the reflection characteristics. For deep hyperthermia (DHT), the most common array setup is a circular or elliptical array of multiple antennas that are matched to the patient by means of a waterbolus filled with (demineralized) water. The use of a waterbolus requires an antenna design that enables efficient radiation from the substrate material of the patch antenna into the bolus water. In the antenna design proposed in this paper, this problem is solved by using water as substrate material, whereby the patch antenna is submerged in the water. This water can be circulated to cool the antenna, thereby increasing the stability of the antenna characteristics at high power. Another advantage is that a water substrate considerably decreases the optimal size of the antenna compared to that of a configuration with a solid substrate $\left(\epsilon_{r}<20\right)$. The drawback of this approach, however, is that the substrate is lossy and its permittivity will vary with the waterbolus temperature, which is determined depending on the skin-cooling strategy required.

Most DHT [6] applicator designs consist of an array of waveguide antennas ("guide fed slot") or dipole antennas [3]. The first designs utilized water-filled waveguides because they are rigid and require no external matching. Despite the utilization of water-filling, these antennas have a very narrow bandwidth and are relatively large and heavy. Another disadvantage of waterfilled waveguides is their relatively strong near-field components [7], [8]. These fields extend to the outside of the waterbolus, especially for low frequencies $(<433 \mathrm{MHz})$, leading to hotspots in superficial tissue effectively reducing penetration depth [7]. The problems with high near-fields were solved by subsequent design utilizing dipole antennas, which are simpler and have a larger bandwidth. Dipoles, however, require an RF-matching network containing a balun [9] to obtain a symmetric radiation field and stable electrical performance. This 


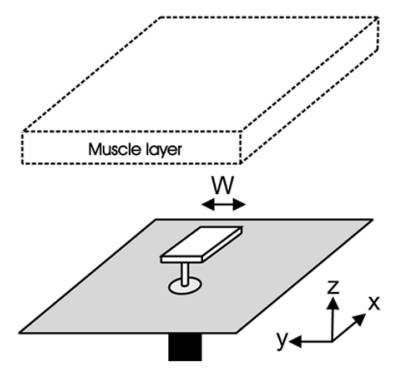

(a)

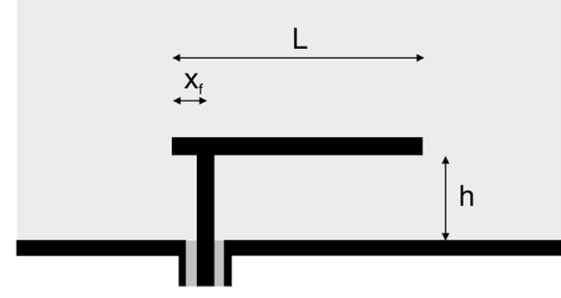

(b)

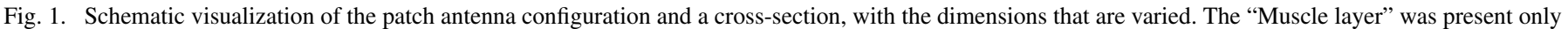
in the setups that were used to investigate the influence of a patient on the reflection characteristics.

matching network absorbs a substantial amount of power and thus decreases the efficiency of the antenna.

The need for such a matching network can be circumvented by using a properly designed probe-fed patch antenna operating within the waterbolus. Since the 1970s, patch antennas have received a lot of attention [10] because they are inexpensive, lightweight, easy to construct and can be made to conform to curved structures. They have also been investigated extensively within the hyperthermia community [4], [11], [12], but mainly for purposes of superficial hyperthermia (SHT) (heating depth $\leq 4 \mathrm{~cm}$ ). For SHT the most important drawback was its small bandwidth and the correspondingly large influence of tissue loadings in the close vicinity of the skin on the antenna's reflection characteristics. For DHT, when a minimal patient-antenna distance can be assured, this influence of tissue loadings is restricted. Further, a highly appealing feature of patch antennas is that they can be fed with a simple coaxial cable, requiring no special matching network between antenna and transmission line. When used for a clinical treatment, antennas in which an RF matching network can be avoided have a clear advantage in terms of reliability and reproducibility. The build-up of heat in the matching network, associated with large mismatches of the antenna load, may cause an unpredictable [13], [14] and unstable behavior of the system.

In this work, we designed and analyzed a patch antenna for use in a head and neck applicator, where the main parameter under investigation was its reflection characteristic. The resulting patch antenna design was analyzed by extensively exploiting 3-D electromagnetic (EM) simulations of various patch antenna setups and measurements. The analysis focussed on the sensitivity of the design to the influences of dimensions (length, width, height, feeding position) and to influences under clinical conditions (water temperature, patient, neighboring patch antennas). The amount of cross-coupling was also estimated using a flat setup. The antenna design was built in an array setting, and its reflection was optimized by fine-tuning its length. Finally, we conducted measurements on multiple versions of these patches to verify the practical feasibility of the design and to check the accuracy of the simulations.

\section{METHODS AND MATERIALS}

\section{A. Design Requirements}

The use of an antenna design within a hyperthermia (HT) applicator leads to some application-specific requirements. The antenna will be embedded in a movable applicator system, hence the antenna should be light, small, and robust. Because E-field components perpendicular to the patient skin lead to high power absorption values at fat-muscle transitions [1], a high $E_{/ /} / E_{\perp}$ ratio at the patient's skin is desired. Furthermore, constructive interference would be optimal for axial electric field components [15]. To reduce power losses in the water bolus $\left(\sigma_{\text {water }} \neq 0[16]\right)$, the antennas should be positioned as close as possible to the patient skin. On the other hand, the distance to the skin has to be large enough to minimize the patient's influence on the reflection characteristics. Further, to avoid an additional matching circuit (extra power losses) which would destroy the initial advantage of the patch antenna, a matched and efficient antenna design was desired. This efficient operation should be possible in the entire range of waterbolus temperatures, which is anticipated to be between $20^{\circ} \mathrm{C}$ and $30^{\circ} \mathrm{C}$ for this semi-deep HT treatment. For the initial design process we anticipated that optimizing the $-15 \mathrm{~dB}$ bandwidth would result in a design with a low sensitivity of the reflection coefficient to different tissue loadings and sufficient room to cope with the change in resonance frequency due to the changes in waterbolus temperature. We aimed to achieve little cross-coupling because it would increase "reflected power" at other antennas, thus virtually decreasing efficiency.

\section{B. Application Specific Configuration}

The patch antenna configuration and its most important dimensions are shown in Fig. 1. The configuration consists of a conducting groundplane with a conducting patch of length $L$ and width $W$ at distance $h$ from the groundplane. The patch is connected, at distance $x_{f}$ from the width of the patch, to the extending conducting rod of a $\mathrm{C}$-female receptacle connector. The diameter of the extending rod is $3 \mathrm{~mm}$ and the diameter of the surrounding Teflon dielectric is $10 \mathrm{~mm}$.

In this configuration, efficient radiation into the waterbolus is obtained by using water as substrate material, i.e., the patch is submerged in the water. The water will be circulated to cool the patient's skin and, at the same time, this circulating water provides cooling of the antenna's self-heating effects at high power $(\sim 100 \mathrm{~W})$. Cross-coupling between antennas is expected to be low in this design because no dielectric transition is present that may enable surface waves. For the first design we used dielectric properties of demineralized water at $433 \mathrm{MHz}$ and $25^{\circ} \mathrm{C}$, i.e., $\epsilon_{r}($ relative permittivity $)=78$ and $\sigma$ (effective conductivity $)=$ $0.04 \mathrm{~S} / \mathrm{m}$. 


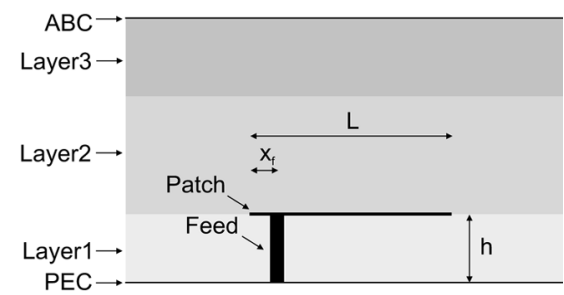

Fig. 2. Schematic visualization of the layered patch setup implementation in Designer. "Patch" is infinitely thin and "Feed" has a diameter of $3.0 \mathrm{~mm}$, both are PEC.

\section{EM Modelling}

1) Ansoft Designer: The design process was initiated by using Ansoft Designer v2.1 [21], a layered 2-D method of moment (MoM) based program. The program makes it possible to define infinitely thin perfectly electrically conducting (PEC) and finite dielectric layers; definition of noninfinite PEC structures and a feed of finite size can also be modelled (Fig. 2). A constrained gradient optimization algorithm was used to optimize the length $L$, width $W$, and distance of the feed to the edge of the patch $x_{f}$ for different heights $h$. To simulate the waterbolus, Layer1 and Layer2 where assigned dielectric properties of demineralized water at $433 \mathrm{MHz}$ [16], [17]. For Layer3, we used dielectric properties of muscle at $433 \mathrm{MHz}$ [18]: $\epsilon_{r}=56, \sigma=$ $0.8 \mathrm{~S} / \mathrm{m}$, to simulate the influence of a patient in the design. Both materials were assumed to be dispersionless. Layer 3 was positioned $50 \mathrm{~mm}$ from the patch, i.e., the thickness of Layer2 was $50 \mathrm{~mm}$.

2) SEMCAD X: The initial design created with Ansoft Designer was further investigated using SEMCAD X v11.0 [22], a commercial finite-difference time-domain (FDTD) [19] based program. For the FDTD algorithm, the entire computational domain must be divided into voxels, i.e., small brick-shaped elements. As SEMCAD X supports a variable grid stepping, grid refinements at small structures can be used to increase the accuracy of the model while retaining acceptable computational times.

The single-patch setup in SEMCAD X is an implementation of the setup of Fig. 1. The combination of connector and connected coaxial cable was modelled using an 8-cm coax with the dimensions of the connector: central conductor (PEC, diameter $=3 \mathrm{~mm})$ Teflon dielectric $\left(\epsilon_{r}=2.08, \sigma=0.462 \mathrm{mS} / \mathrm{m}\right)$. Because modelling of this connection had a considerable impact on the results, we used the most accurate model. Four voltage sources were positioned in the coax, each in a different quadrant, $4 \mathrm{~cm}$ beneath the ground plane. These sources were excited with a Gaussian pulse modulated with a 433-MHz sinusoid containing frequencies between 350 and $500 \mathrm{MHz}$. The resulting E-field pattern in the coax was investigated to ensure that the correct mode was propagating towards the patch. An infinite water environment was modelled by using four or eight layers of Berenger's perfect matched layers (PML) [19] as absorbing boundary conditions $(\mathrm{ABC})$. To extract the energy propagating away from the antenna, eight layers of PML were used at the truncation of the coaxial cable. The size of the computational domain was $20 \mathrm{~cm} \times 20 \mathrm{~cm} \times 22 \mathrm{~cm}$ (length $\times$ width $\times$ height), i.e., the distance from the patch to the $\mathrm{ABC}$ was always more than $8.8 \mathrm{~cm}\left(>\lambda_{\text {water }}\right)$. The setup was modelled with grid-steps from $3.2 \mathrm{~mm}$ down to $0.06 \mathrm{~mm}$. Refinement down to $0.06 \mathrm{~mm}$ at the patch and feed proved necessary because the model appeared quite sensitive to accurate modelling of the coaxial cable. Further the grid-steps at the patch were always smaller than $0.5 \mathrm{~mm}$. This resulted in a total amount of 3.6 million voxels. Voltages and currents were recorded in the coaxial cable at the location of the ground plane from which the S-parameters were calculated. Transient simulations were terminated after $\sim 55 \mathrm{~ns}$ when signals had sufficiently decayed $(<0.01 \%)$, while for harmonic simulations $(f=433 \mathrm{MHz}) 10$ periods were simulated.

As in Designer, the water of the waterbolus was modelled by using dielectric properties of water at $25^{\circ} \mathrm{C}$ and muscle $\left(\epsilon_{r}=\right.$ $57, \sigma=0.8 \mathrm{~S} / \mathrm{m}$ ) at $433 \mathrm{MHz}$, i.e., both materials were assumed to be dispersionless. The influence of a different waterbolus temperatures was investigated by using dielectric properties of water between $20^{\circ} \mathrm{C}$ and $30^{\circ} \mathrm{C}$ [16], [17]. Variations of the reflection coefficient due to patient distance was investigated by positioning the muscle layer (Fig. 1) at various distances from the patch antenna. The dielectric properties of this layer were varied as well in addition to investigate the influence of different patients.

3) Multiple Antenna Setup: To investigate the influence of neighbor antennas and the amount of cross-coupling, we created setups with two antennas that were always oriented in the same direction. Three arrangements of two antennas were investigated, i.e., horizontal (H-plane), diagonal and vertical (E-plane). The distance between the antennas was always $10 \mathrm{~cm}$, since this is the worst case distance of the anticipated twelve antennas on a 40-cm diameter ring [20]. The combined influence of a patient was investigated by positioning a muscle layer at $\infty$ (muscle layer absent), $10 \mathrm{~cm}$ or $5 \mathrm{~cm}$ for all arrangements. The setups were also used to determine the amount of cross-coupling $\left(S_{12}\right)$ between two neighbors. Hereto we recorded voltages and currents simultaneously at both primary and secondary antenna to be able to calculate $S_{11}$ and $S_{12}$ parameters.

\section{Clinical Antenna Setup: Measurements and Simulation}

In previous publications [2], [20] we have shown that the optimum setup for an $\mathrm{H} \& \mathrm{~N}$ hyperthermia applicator is a circular array (diameter $=40 \mathrm{~cm}$ ) of two rings of six antennas (spacing between rings $=6 \mathrm{~cm}$ ). We constructed such a setup to measure the reflection characteristics of the patch antenna design within the applicator, and as such validate our theoretical analysis. Fig. 3 shows the measurement setup, which consisted of a PEC cylindrical backplane $($ diameter $=40 \mathrm{~cm}$, height $=15 \mathrm{~cm})$ that formed the groundplane of the patch design. To decrease the influence of a water-air transition, this cylinder was positioned on top of an absorbing flat muscle phantom, which was filled with demineralized water $\left(25^{\circ} \mathrm{C}\right)$ to model the waterbolus. A cylinder (diameter $=88 \mathrm{~mm}$ ) filled with a $10 \mathrm{~g} / \mathrm{l}$ saline solution was used to model the patient. The theoretical patch design was adapted somewhat to increase its robustness under clinical conditions. Hereto we added a low-dielectric support which was only $10 \mathrm{~mm}$ in the $x$-direction and was positioned centrally under the patch to avoid influences on radiation pattern and reflection coefficient. By simulations we found that a support that is both centrally placed and sufficiently small has 


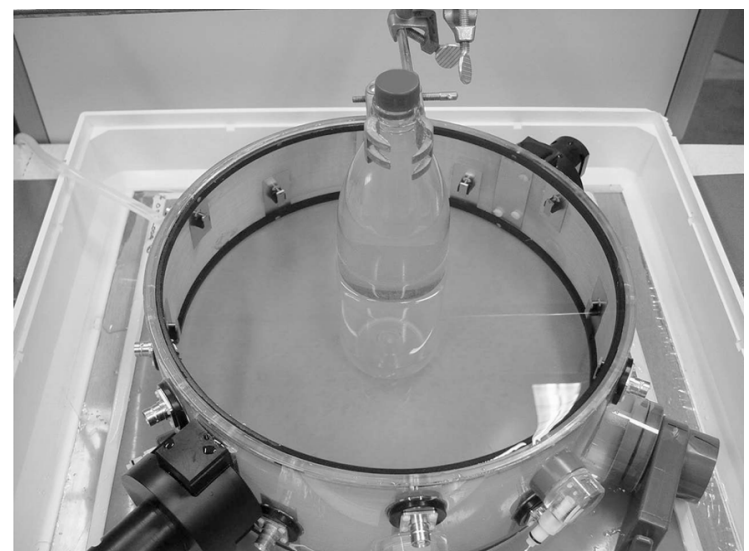

Fig. 3. Picture of the measurement setup with 12 antennas at staggered locations in two rings [20]. Also visible are 1) the centrally placed bottle containing saline water, 2) the gauze that forms the conducting backplane (limitedly visible) and separate conducting backplanes per antenna, and 3) the flat muscle phantom on which the antenna ring is positioned. The reflection characteristics of the lower six antennas were measured for validation of the model.

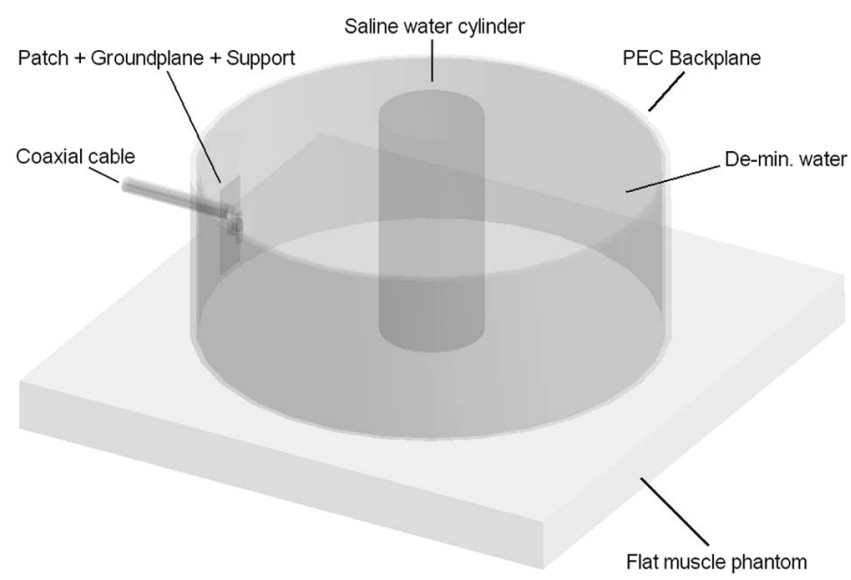

Fig. 4. Solid model in SEMCAD X of the measurement setup.

a negligible influence on the resonance frequency. For the measurements we used the six antennas of the lower ring $(4.5 \mathrm{~cm}$ above the flat muscle phantom) since these are affected the least by the water-air transition that 1) in practice is further away due to bulging of the waterbolus and 2) decreases the reproducibility and predictability of the setup. Reflection measurements were performed using a 8751A network analyzer (Agilent Technologies, USA). Using the results of these measurements, the lengths of the patches were adapted one final time.

Fig. 4 shows the corresponding solid model implementation for one antenna of the measurement setup in SEMCAD X. By using the model settings described earlier, a model that consisted of $6 \mathrm{M}$ cells was obtained, and total simulation time was $25 \mathrm{~h}$ using a special hardware accelerator (aXware [22]). Due to memory limitations, we modelled only one antenna.

\section{RESULTS}

\section{A. Antenna Design}

Using Designer, we found that a resonant antenna was obtained only at heights ( $h$ : substrate thickness) over $5 \mathrm{~mm}$ and lengths much smaller than $\lambda_{\text {water }} / 2$. Using Designer's gradient

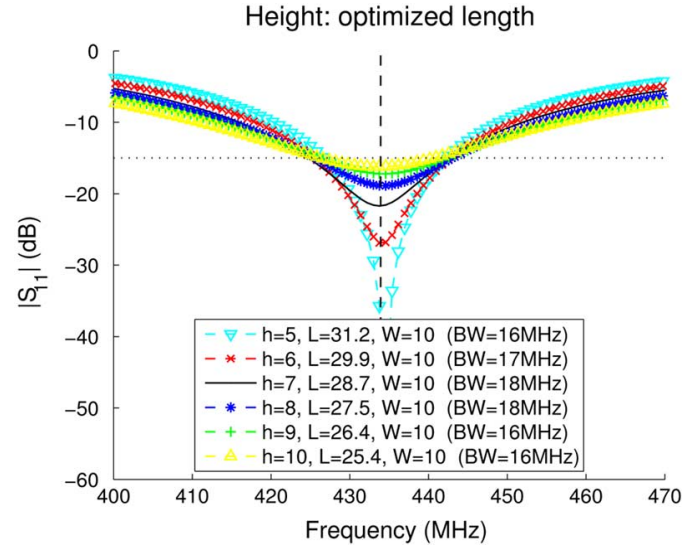

Fig. 5. Reflection characteristics calculated with SEMCAD X for various patch heights and optimized lengths.

optimizer, we optimized the patch dimensions for substrate thicknesses between 5 and $10 \mathrm{~mm}$. The optimization procedure provided best results for setups with patch heights between approximately 8 and $9 \mathrm{~mm}$. However, all minimum reflection values were well beneath $-20 \mathrm{~dB}$, and the $-15 \mathrm{~dB}$ bandwidth was $\sim 28 \mathrm{MHz}(6.5 \%)$, and thus sufficient for our purposes. The influence of the presence of a muscle layer (Fig. 2: Layer3) at a minimum distance of $4 \mathrm{~cm}$ from the antenna's groundplane was found to be negligible, i.e., it resulted in the same optimal dimensions as in the optimization without the muscle layer. We chose to continue our investigations using a setup of: $L=$ $27.8 \mathrm{~mm}, W=10 \mathrm{~mm}, h=9 \mathrm{~mm}, x_{f}=1.75 \mathrm{~mm}$.

1) Semcad X: When implementing the Ansoft Designer (2-D model) optimized setup ( $h=9 \mathrm{~mm}$ ) in SEMCAD X v10.0 (3-D model), we found that Designer overestimated the performance in terms of reflection characteristics. Using SEMCAD X, we fine-tuned the setup for better reflection characteristics. Hereto we investigated setups with heights around $h=9 \mathrm{~mm}$, and scaled the lengths $L$ of these setups to find a resonant antenna at $433 \mathrm{MHz}\left(W=10 \mathrm{~mm}, x_{f}=1.75 \mathrm{~mm}\right)$. The results of this optimization are shown in Fig. 5. Here, we selected the setup with $h=7 \mathrm{~mm}$ as optimum, since this yielded the highest bandwidth $(18 \mathrm{MHz}=4.2 \%)$.

Subsequently, we analyzed the sensitivity of the design to its design parameters. This is useful in assessing the influence of construction errors and for selecting the best dimension for fine-tuning the clinical antenna. Fig. 6 shows the sensitivity of the selection to all four design parameters. This figure shows that variations in height and length are of great importance: the resonance frequency changes in a rather linear fashion by approximately $12 \mathrm{MHz} / \mathrm{mm}$ (length) and $15 \mathrm{MHz} / \mathrm{mm}$ (height). Small realistic errors in the location of the feed had little influence. Changing the width only scaled the real part of the antenna's impedance, i.e., the resonance frequency remained unchanged. Therefore, we could further improve the design by using the width that corresponded with a real part of the impedance closest to $50 \Omega$ at $433 \mathrm{MHz}$. The final theoretical design was thus set at: $L=28.7 \mathrm{~mm}, W=8 \mathrm{~mm}, h=7 \mathrm{~mm}$, $x_{f}=1.75 \mathrm{~mm}$.

The electric field for this optimized setup without ("all water") or with ("muscle at $4 \mathrm{~cm}$ ") a patient is shown in Fig. 7. 

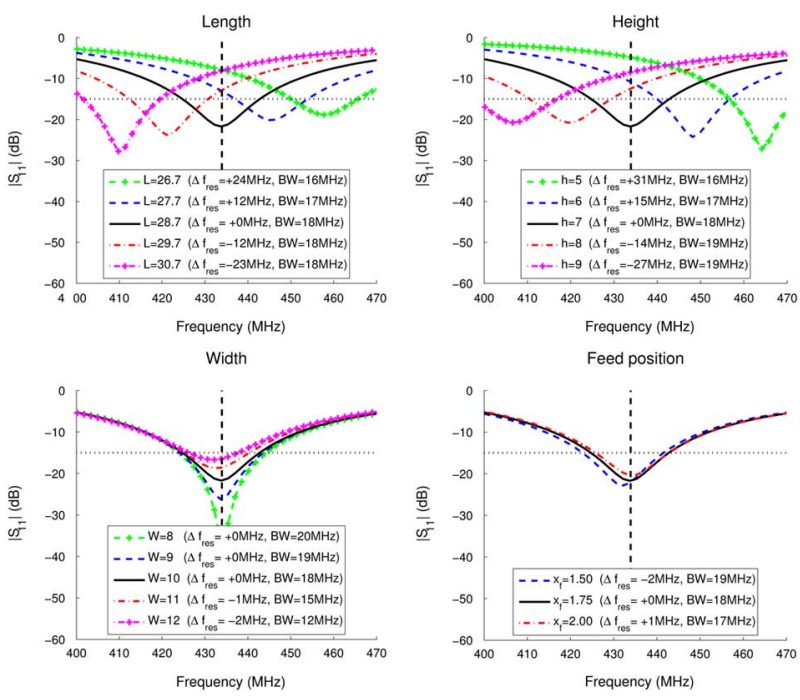

Fig. 6. Sensitivity of the antenna design $(L=28.7 \mathrm{~mm}, W=10.0 \mathrm{~mm}, h=$ $7.0 \mathrm{~mm}$, and $x_{f}=1.75 \mathrm{~mm}$ ) to antenna dimensions, i.e., length, height, width, and feed position $\left(x_{f}\right)$, calculated by SEMCAD X.

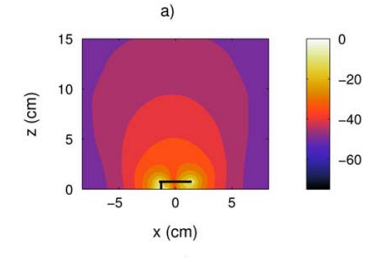

c)

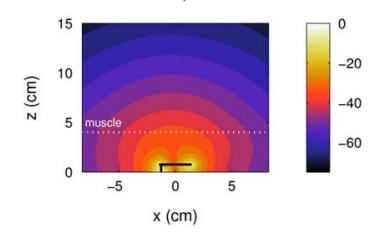

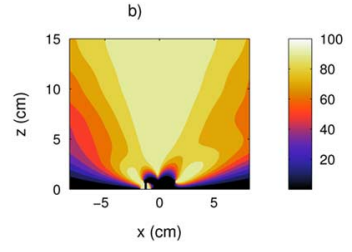

d)

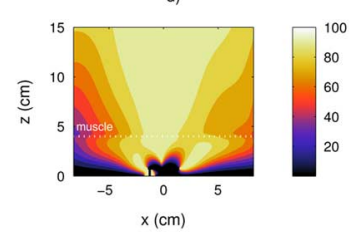

Fig. 7. Rms value of the electric field (a), (c) and respective amount of co-polarization (b), (d) corresponding to a patch in water without (a), (b) or with (c), (d) a muscle layer. Cross-sections through $y=0$ are shown and the water-muscle transition is indicated by a white dotted line.

In this figure, we visualize the rms value of the electric field and the amount of co-polarization, both with and without a patient. The figures show that the electric field decay is faster in muscle (a), (c). The amount of co-polarization, however, is high $(-5 \mathrm{~cm}<x<5 \mathrm{~cm}:>80 \%)$ for both the setups investigated (b), (d).

\section{B. Characteristics of the Design Under Clinical Conditions}

To assess the performance of the design under clinical conditions, we theoretically analyzed its reflection characteristics for 1) waterbolus temperature changes, 2) the presence of a patient, and 3) the presence of a neighbor antenna. Fig. 8 shows the reflection curves corresponding to water temperatures between $15^{\circ} \mathrm{C}$ and $35^{\circ} \mathrm{C}$. For increasing temperatures, these curves show that the shape of the reflection curve remains intact, but that the increase in resonance frequency was linear at around $1 \mathrm{MHz} /{ }^{\circ} \mathrm{C}$. Fig. 9 shows the reflection curves corresponding to decreasing patient-antenna distances, i.e., decreasing muscle layer heights. Different patient-antenna distances $\left(D_{m}>4 \mathrm{~cm}\right)$

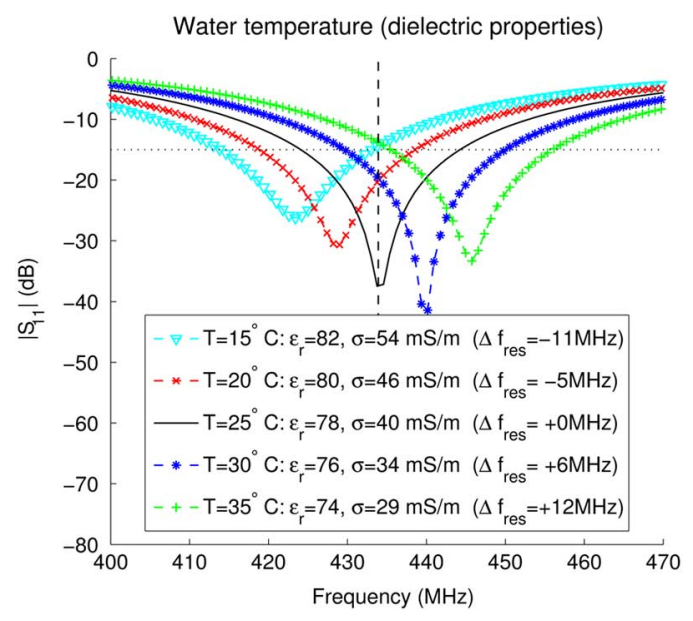

Fig. 8. Sensitivity of the reflection coefficient to changes in waterbolus temperature.

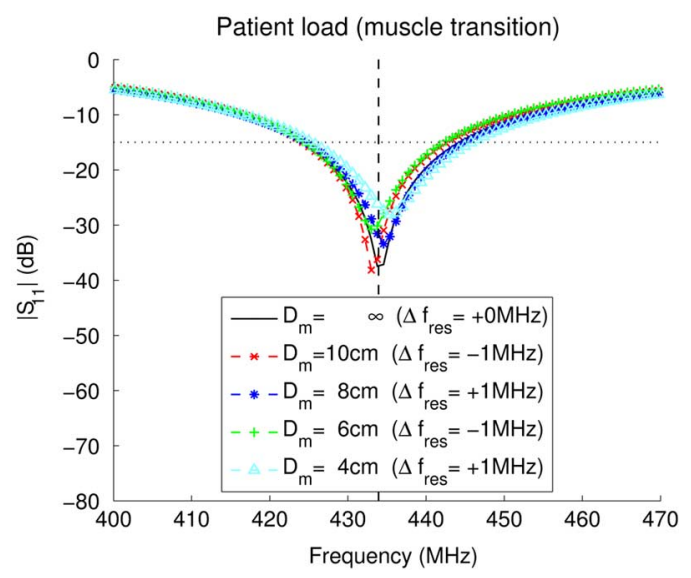

Fig. 9. Sensitivity of the reflection coefficient to an approaching muscle layer. $D_{m}$ is the distance between groundplane and muscle transition.

showed little influence, i.e., an almost stable $(<1 \mathrm{MHz})$ reflection pattern. An extreme $(50 \%)$ decrease and increase of the dielectric properties of the muscle layer $\left(D_{m}=5 \mathrm{~cm}\right)$ resulted only in a very small additional alteration of maximum $1 \mathrm{MHz}$ in resonance frequency. The combined influence of a patient and a neighbor antenna was investigated for three setups: 1) without a muscle layer, 2) with a muscle layer at $10 \mathrm{~cm}$, and 3) with a muscle layer at $5 \mathrm{~cm}$. The predictions show $1 \mathrm{MHz}$ maximum, uncorrelated, differences in resonance frequency with respect to the reference situation: a setup with no muscle layer and no neighbor antenna. All orientations (vertical, horizontal, and diagonal) and all muscle distances $(\infty, 10 \mathrm{~cm}$, and $5 \mathrm{~cm})$ show little cross-coupling between neighbors: $S_{21}<-27 \mathrm{~dB}$.

\section{Clinical Antenna Setup: Measurements and Simulation}

Utilizing measurements of patch antennas in the applicator setup (see Fig. 4), we made a final amendment to the theoretical design, i.e., we reduced the length: $L=28.2 \mathrm{~mm}$, $W=8 \mathrm{~mm}, h=7 \mathrm{~mm}, x_{f}=1.75 \mathrm{~mm}$. Fig. 10 shows the curves of the average measured reflection characteristics of six of such patch antennas, while the water temperature was $24.8^{\circ} \mathrm{C}$. The figure indicates that, on average, the patches had the following characteristics: $S_{11}(433 \mathrm{MHz})=-21 \mathrm{~dB}$ 


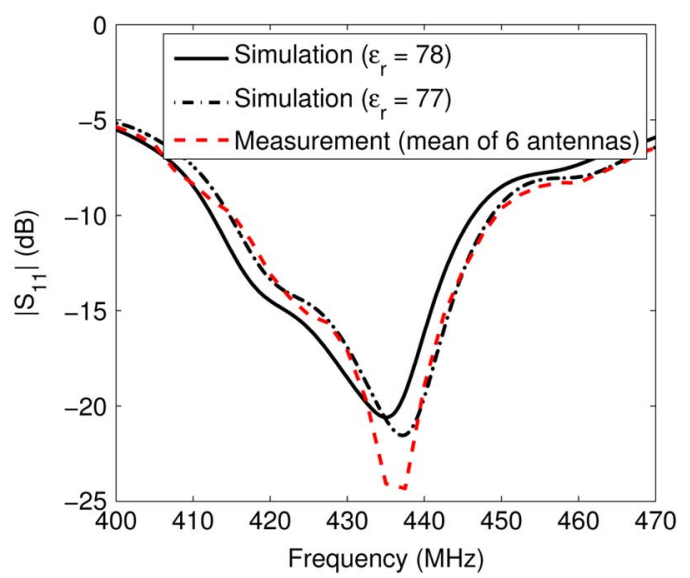

Fig. 10. Mean of reflection measurements of six patches in the applicator ring and corresponding FDTD simulation results for two values for the permittivity of the water in the waterbolus.

and $\mathrm{BW}=18.5 \mathrm{MHz}(4.3 \%)$. This figure also shows a good agreement between simulated values and measurements. For these calculations we used as permittivity both $\epsilon_{r}=78$ and $\epsilon_{r}=77$, to illustrate the slight better agreement at this lower value. The figure also shows that the model can be used to predict the location of the resonance frequency with reasonable accuracy but the predicted bandwidth is somewhat lower $(\mathrm{BW}=17.1 \mathrm{MHz})$. Since the depth of the peak depends on the conductivity of the water, this might explain the discrepancy in depth of the resonance peak remaining $(\sim 4 \mathrm{~dB})$. We expect that, in the more predictable flat setup that we used for the analysis, differences will be even less than in this applicator setup with its more cavity-like behavior.

\section{DISCUSSION AND CONCLUSION}

In this study, we have designed a patch antenna and when we examine its characteristics against the design criteria formulated at the start, we can draw the following conclusions: 1) the patch antenna design has a high amount $(>80 \%)$ of co-polarization in broadside direction; 2) while the dependence of the resonance frequency is linearly dependent on the temperature, it can be chosen to allow waterbolus temperatures between $15^{\circ} \mathrm{C}$ and $35^{\circ} \mathrm{C}$; 3) the influence of a patient at a distance as close as $4 \mathrm{~cm}$ from the groundplane is a maximum resonance frequency shift of $1 \mathrm{MHz}$; and 4) cross-coupling with neighbor antennas in all investigated realistic setups was predicted to be lower than $-27 \mathrm{~dB}$. Although these low cross-coupling values indicate that it will be of no concern in the clinical applicator, this aspect will be investigated further; we are specifically interested in the combined cross-coupling when all 12 antennas are operating coherently.

A clinical version of the patch antenna was constructed, and modifications were made for better mechanical robustness and to conform it within an array of antennas. The simulations of the theoretical setup were validated by performing measurements with six of these patch antennas. These measurements show a very good agreement for the resonance frequencies obtained, and a good agreement for the obtained bandwidths. We found that the length of the patch antenna for resonance in an infinite water halfspace $\left(L_{\mathrm{res}}=28.7 \mathrm{~mm}\right)$ could be slightly reduced for resonant behavior within the applicator $\left(L_{\mathrm{res}}=28.2 \mathrm{~mm}\right)$. The situation in the applicator differs from the theoretical situation because 1) the groundplane was curved, 2) a low-permittivity support was added for stability, and 3) the patch was placed in the waterbolus, which acts to some extent as a cylindrical resonant cavity. Although the contribution of each effect to this decrease in required length is unclear to us, the adapted patch length was accurately predicted by SEMCAD X. We further found that the bandwidth obtained with the infinite layered setup (20 MHz, $4.6 \%$ ) is somewhat larger than that in the applicator setup, i.e., simulation: $17.1 \mathrm{MHz}(3.9 \%)$ and measurement: $18.5 \mathrm{MHz}$ (4.3\%). This $-15 \mathrm{~dB}$ bandwidth is similar compared to a probe-fed patch design radiating into air as reported by Garg et al. [10].

Dispersionless materials were assumed for simulations throughout this paper, i.e., we used properties of muscle and water at $433 \mathrm{MHz}$. Theoretically, in our entire frequency range (400-470 MHz), the variation in permittivity values is small: $-0.01 \%$ for demineralized water [16] and $-0.9 \%$ for muscle [18]. The variation in conductivity, however, is much greater: $+28 \%$ for demineralized water [16] and $+2.3 \%$ for muscle [18]. On the basis of our own measurements with saline water (unpublished data), we know that the conductivity of the water has an effect on the depth of the $S_{11}$ curve of a patch antenna, i.e., increasing conductivity leads to a flatter curve, but not on the resonance frequency. By comparing the measurements with the simulations (Fig. 10), we also show that the main difference is indeed the depth of the resonance peak, but that the estimation of the resonance frequency is fairly accurate $\left(\Delta f_{\text {res }} \sim 2 \mathrm{MHz}\right)$.

We have investigated the application of a probe-fed patch antenna design in a phased-array $\mathrm{H} \& \mathrm{~N}$ hyperthermia applicator. If we convert the results of our analysis to the situation for DHT, this design has several advantages over the designs currently used. The main advantage of this design is the direct feeding with a coaxial cable, i.e., no matching circuit is required. As we explained before, this leads to easier and more accurate modelling, and also to greater robustness and higher efficiency. A drawback of the design for a more broadband application (multiple frequencies) is its relatively small bandwidth. However, in this application this is not a problem, as we use only a single frequency.

\section{ACKNOWLEDGMENT}

The authors would like to thank T. Bertuch and H. Strijbos for their help on the initial patch antenna design.

\section{REFERENCES}

[1] M. M. Paulides, D. H. M. Wielheesen, J. Van der Zee, and G. C. Van Rhoon, "Assessment of the local SAR distortion by major anatomical structures in a cylindrical neck phantom," Int. J. Hyperthermia, vol. 21, pp. 125-140, 2005.

[2] M. M. Paulides, S. H. J. A. Vossen, A. P. M. Zwamborn, and G. C. Van Rhoon, "Theoretical investigation into the feasibility to deposit RF energy centrally in the head and neck region," Int. J. Rad. Onc. Biol. Phys., vol. 63, pp. 634-642, 2005.

[3] P. R. Stauffer, "Evolving technology for thermal therapy of cancer," Int. J. Hyperthermia, vol. 21, pp. 731-44, 2005.

[4] J. W. Hand and J. R. James, Physical Techniques in Clinical Hyperthermia. Letchworth, U.K.: Research Studies Press, 1986, ch. 4. 
[5] R. W. P. King, B. S. Trembley, and J. W. Strohbein, "The electromagnetic field of an insulated antenna in a conducting or dielectric medium," IEEE Trans. Microw. Theory Tech., vol. MTT-31, no. 7, pp. 574-583, Jul. 1983.

[6] J. Van der Zee, D. G. Gonzalez, G. C. Van Rhoon, J. D. Van Dijk, W. L. van Putten, and A. A. Hart, "Comparison of radiotherapy alone with radiotherapy plus hyperthermia in locally advanced pelvic tumours: A prospective, randomised, multicentre trial," Dutch Deep Hyperthermia Group Lancet, vol. 1; 355, pp. 1119-1125, 2000.

[7] P. Nilsson, "Physics and technique of microwave-induced hyperthermia in the treatment of malignant tumours," Ph.D. dissertation, Lund Univ., Lund, Sweden, 1984.

[8] G. C. Van Rhoon, "Radiofrequency hyperthermia systems: Experimental and clinical assessment of the feasibility of radiofrequency hyperthermia systems for loco-regional deep heating," Ph.D. dissertation, Delft Univ., Delft, The Netherlands, 1994.

[9] C. A. Balanis, Antenna Theory: Analysis and Design, 2nd ed. New York: Wiley, 1997, ch. 4.

[10] R. Garg, P. Bhartia, I. Bahl, and A. Ittipiboon, Microstrip Antenna Design Handbook. Norwood, MA: Artech, 2001.

[11] H. R. Underwood, A. F. Peterson, and R. L. Magin, "Electric-field distribution near rectangular microstrip radiators for hyperthermia heating: Theory versus experiment in water," IEEE Trans. Biomed. Eng., vol. 39, no. 2, pp. 146-153, Feb. 1992.

[12] C. Michel, P. Y. Cresson, L. Dubois, M. Chive, and J. Pribetich, "Design and modeling of microstrip-microslot applicators with several patches and apertures for microwave hyperthermia," Microw. Opt. Tech. Lett., vol. 14, no. 2, pp. 121-126, 1997.

[13] P. Wust, M. Seebas, J. Gellerman, P. Deuflhard, and J. Nadobny, "Antenna arrays in the SIGMA-eye applicator: Interactions and tranforming networks," Med. Phys., vol. 28, pp. 1793-1805, 2001.

[14] J. Nadobny, H. Fähling, M. J. Hagmann, P. F. Turner, W. Wlodarczyk, J. M. Gellermann, P. Deuflhard, and P. Wust, "Experimental and numerical investigation of feed-point parameters in a 3-D hyperthermia applicator using different FDTD models of feed networks," IEEE Trans. Biomed. Eng., vol. 49, no. 11, pp. 1348-1359, Nov. 2002.

[15] S. B. Field and C. Franconi, Physics and Technology of Hyperthermia. Dordrecht, The Netherlands: Martinus Nijhoff, 1987, ch. I-7.

[16] A. Stogryn, "Equations for calculating the dielectric constant of saline water (correspondence)," IEEE Trans. Microw. Theory Tech., vol. 19, no. 8, pp. 733-736, Aug. 1971.

[17] J. J. W. Lagendijk, P. M. Van den Berg, J. W. Hand, N. K. Uzunoglu, R. Sheppard, J. B. Andersen, F. Bardati, and J. C. Bolomey, "Treatment planning and modelling in hyperthermia," Postgraduate School of Medical Physics, II Univ. Rome, Rome, Italy, Task Group Report, 1992.

[18] S. Gabriel, R. W. Lau, and C. Gabriel, "The dielectric properties of biological tissues III: Parametric models for the dielectric spectrum of tissues," Phys. Med. Biol., vol. 41, pp. 2271-2293, 1996.

[19] A. Taflove and S. C. Hagness, Computational Electrodynamics: The Finite-Difference Time-Domain Method, 3rd ed. Norwood, MA: Artech, 2005.

[20] M. M. Paulides, J. F. Bakker, A. P. M. Zwamborn, and G. C. Van Rhoon, "A head and neck hyperthermia applicator: Theoretical antenna array design," Int. J. Hyperthermia, vol. 23, no. 1, pp. 59-67, 2007.

[21] Ansoft Designer. Ansoft Corp., Pittsburgh, PA [Online]. Available: http://www.ansoft.com

[22] SEMCAD: The Simulation platform for EMC, Antenna Design and Dosimetry. Schmid \& Partner Engineering AG, Zurich, Switzerland [Online]. Available: http://www.semcad.com

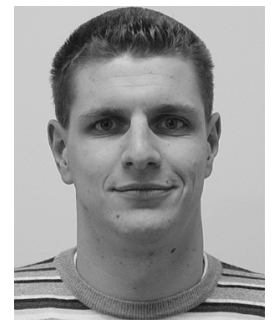

Margarethus M. Paulides was born in The Netherlands in 1979. He received the M.Sc. degree in electrical engineering from the Eindhoven University of Technology, Eindhoven, The Netherlands, in 2002. He is currently working towards the Ph.D. degree in the Department of Radiotherapy, Erasmus MC-Daniel den Hoed Cancer Center, Rotterdam, The Netherlands.

His work is primarily on a Dutch Cancer Society project entitled: "Development, construction and clinical testing of a novel electromagnetic applicator for hyperthermia treatment of advanced head and neck carcinoma". His research interests are in the area of hyperthermia treatment planning, bioelectromagnetics, antenna design, and applied clinical physics.

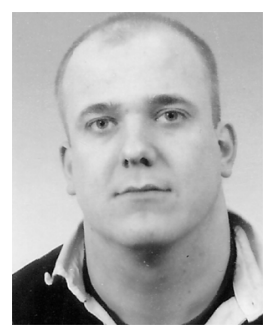

Jurriaan F. Bakker was born in The Netherlands in 1979. He received the B.Eng. degree in engineering physics from Rijswijk University of Professional Technical Education, Rijswijk, The Netherlands, in 2003. He is currently working towards the M.Sc. degree in engineering physics at Delft University of Technology, Delft, The Netherlands.

Further, he is working on a Dutch Cancer Society project entitled: "Development, construction and clinical testing of a novel electromagnetic applicator for hyperthermia treatment of advanced head and

neck carcinoma".

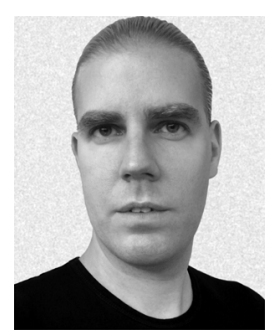

Nicolas Chavannes (S'94-M'98) was born in Bern, Switzerland, in April 1972. He received the M.S. and $\mathrm{Ph} . \mathrm{D}$. degrees in electrical engineering from the Swiss Federal Institute of Technology (ETH), Zurich, Switzerland, in 1998 and 2002, respectively.

In 1996, he joined the Bioelectromagnetics/EMC Group (BIOEM/EMC) at ETH Zurich where he was involved in computational electrodynamics and related dosimetric applications. From 1998 to 2002, he was with the Laboratory for Electromagnetic Fields and Microwave Electronics (IFH) as well as the Laboratory for Integrated Systems (IIS), both located at ETH Zurich. There, his research activities were focused on the development of FDTD local refinement techniques and their application to numerical near-field analysis. In late 1999, he joined the Foundation for Research on Information Technologies in Society (IT'IS), Switzerland, where he is currently in charge of the development and extension of a simulation platform targeted for antenna modeling and MTE design in complex environments, dosimetry and optics applications. In early 2002, he joined Schmid \& Partner Engineering AG (SPEAG), Zurich, as Director of the software R\&D team. His primary research interests include the development, implementation and application of computational modeling and simulation techniques to electromagnetics in general, and antennas as well as bioelectromagnetic interaction mechanisms in particular.

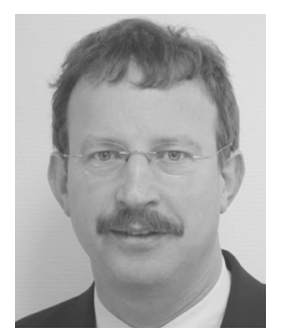

Gerard C. van Rhoon (M'98) received the Ph.D. degree from the Delft University of Technology, Delft, The Netherlands, in 1994.

He leads the research program of the hyperthermia Unit of the Erasmus MC-Daniel den Hoed Cancer, which focuses on development of the technology needed for the clinical application of hyperthermia. $\mathrm{He}$ is Secretary-General of the European Society for Hyperthermic Oncology. He is a member of the Dutch Health Council and involved in environmental health topics concerning the public use of electromagnetic fields for telecommunication and power-lines. 